\title{
Prediction of Particle Size Behaviour near Impingement Wall
}

\author{
Eid S. Alatawi \\ KACST \\ King Rd., Riyadh, Saudi Arabia \\ alatawi@kacst.edu.sa
}

\section{Extended Abstract}

This study presents a numerical prediction of the particle size behaviour near the wall of an impinging jet with nozzle-to-surface distance, $\mathrm{L} / \mathrm{D}=2$. The continuous phase flow was solved using Reynolds-averaged Navier Stokes (RANS) along with Shear Stress Transport turbulence model (SST). The particulate phase was simulated using a one-way coupling Lagrangian random-walk eddy-interaction model (EIM) implemented in an in-house FORTRAN code, where the equation of motion of particles was solved numerically using $4^{\text {th }}$ order Rung-Kutta method. The main forces working on the solid particles were the drag force and the gravity force. Three different particle sizes of 5, 10, $20 \mu \mathrm{m}$ were used in the simulation. In EIMs [1], one particle is allowed to interact successively with various eddies. Each eddy has a characteristic lifetime, length, and velocity scales obtained from the single-phase flow calculation results. The end of the interaction between the particle and one eddy occurs when the lifetime of the eddy is over or when the particle crosses the eddy. At this instant, a new interaction with the particle and a new eddy is started. The particle will have another trajectory according to its equation of motion [2] and [3]. In order to obtain velocity or deposition statistics, hundreds of particles must be released into the flow.

In the present study, for the time scale or the eddy life time is maintained constant during one eddy particle interaction. However, the eddy characteristic velocity scale (known as eddy velocity) is varying during the interaction. The eddy velocity is obtained by multiplying, the root-mean-square (RMS) fluid fluctuating velocity by a random number, $N$, generated from a Gaussian probability density function of zero mean and unity standard deviation. The fluid velocity components in the equation of motion of particles are instantaneous quantities composed of the mean part and the fluctuating part. The fluctuating part of the instantaneous fluid velocity is obtained through modelling. Therefore, three local fluctuating velocities are computed for each eddy at the start of one eddy-particle interaction.

In this study, airflow at $25 \mathrm{C}^{\circ}$ incompressible and steady state fluid flow was assumed in the solution. Inlet conditions consisted of a top hat profile $(\mathrm{u}=10.5 \mathrm{~m} / \mathrm{s})$, a turbulence intensity of $5 \%$ of the mean velocity and a turbulence length scale of $10 \%$ of the inlet diameter $(15 \mathrm{~mm})$. The Reynolds number based on the inlet diameter is $\operatorname{Re}=\rho u D / \mu=$ 10000 , where the air density is $\rho=1.18 \mathrm{~kg} / \mathrm{m}^{3}$ and $\mu$ is the viscosity of the fluid $\left(\mu=1.824 \times 10^{-5} \mathrm{~kg} / \mathrm{m} . \mathrm{s}\right)$. A constant zero gage pressure was applied at the outlet opening of the computational domain. In addition, no slip condition was applied on the impingement and side walls.

The results showed that the smallest particles $(5 \mu \mathrm{m})$ behave closely as the streamline of the fluid phase due to its small inertia effect. However, for the largest particles $(20 \mu \mathrm{m})$, they deposit on the wall immediately as it enters the stagnation region, due to their large size, in different behaviour from the $5 \mu \mathrm{m}$-particles. The $10 \mu \mathrm{m}$-particles show intermediate behaviour between the two sizes, although its tendency to the $20 \mu \mathrm{m}$-particle size behaviour.

\section{References}

[1] A.D. Gosman and E. Ioannides, "Aspects of Computer Simulation of Liquid-fueled Combustors," in AIAA 19th Aerospace Sci. Meeting, 1981, vol. 8.

[2] S. Elgobashi and W.A. Sirignano, "On the Equation for Spherical-Particle Motion: Effect of Reynolds and Acceleration Numbers", Journal of Fluid Mechanics, vol. 367, pp. 221-253, 1998.

[3] R. Mei, "Velocity fidelity of flow tracer particles," Exp. Fluids, vol. 22, pp. 1-13, 1996. 